\title{
A 3-Bit 10-MSps Low Power CMOS Flash ADC
}

\author{
Andrew Selasi Agbemenu \\ Dept. of Computer Eng. \\ KNUST \\ Kumasi, Ghana
}

\author{
Ernest Ofosu Addo \\ Dept. of Info. Eng. and Math. \\ University of Siena \\ Siena, Italy
}

\author{
Benjamin Kommey \\ Dept. of Computer Eng. \\ KNUST \\ Kumasi, Ghana
}

\begin{abstract}
Flash ADCs employ multiple comparator circuits to introduce parallelism in data conversion. Having their speed limited only by transistor gate and comparator propagation delay, flash converters have the fastest signal conversion speeds amongst all ADC architecture implementations. This paper details the design of a low power 3-bit flash ADC with a $3 \mathrm{~V}$ supply realised in $0.6 \mathrm{mi}-$ cron CMOS technology. The designed ADC exhibits a voltage resolution of $34.13 \mathrm{mV}$ and draws $23.88 \mathrm{~mW}$ power at $2.17 \mathrm{MHz}$.
\end{abstract}

\section{General Terms}

Flash ADC, CMOS

\section{Keywords}

Resistive ladder, Comparators, Bubble noise suppression, Hysteresis

\section{INTRODUCTION}

Applications of flash ADCs are widespread in the areas ranging from satellite communication to radar processing where large bandwidth is essential for optimum system operation [7, 6]. The fast nature of these ADCs arise from the parallel comparison of input signals and the high input time quantization speed. This sampling speed is typically limited only by comparators and the digital logic switching. The fundamental performance of these converters is however heavily dependent on the characteristics of their comparators and voltage ladders. Errors in these constituent blocks consequently have severe effects on conversion properties and abilities. With $2^{n-1}$ comparators, $2^{n}$ resistors, and other logic circuits required in a typical n-bit flash converter for operation, these ADCs are naturally plagued with large power and area liabilities.

Flash converters are also limited to low resolutions as the hardware needed, doubles per resolution increase. Power dissipation in this case also increases by more than a factor of two [9] 7]. Non-idealities in converter building blocks can therefore not be tolerated and must be reduced as much as possible. This work explores the use of hysteresis and other techniques in the design of the constituting blocks of a flash ADC to address common converter error sources such as metastability, bubble error, and substrate noise.

This paper is organized as follows. Section 2 discusses the flash architecture, Section 3 discusses the proposed design. Section 4 presents the post layout results and concludes with Section 5

\section{FLASH CONVERTER ARCHITECTURE}

The highest potential sample rate characteristic of the flash ADC arises from the rather parallel fashion in which a single analog input signal, $V_{I N}$ is compared to the converter's reference levels to decide correct quantization levels[6]. The 7 reference levels, shown in Figure 1 are typically generated through a resistor ladder where 8 equally valued resistors divide the ADC's applied
Table 1. : Design parameters of proposed flash ADC

\begin{tabular}{|l|l|}
\hline Parameter & Description \\
\hline Bandwidth & $2 \mathrm{MHz}$ \\
\hline Comparator propagation delay & $3.2 \mathrm{~ns}$ \\
\hline Voltage resolution & $35 \mathrm{mV}$ \\
\hline ADC power consumption & $30 \mathrm{~mW}$ \\
\hline
\end{tabular}

reference voltage, $V_{R E F}$. Seven comparators are then used to establish a comparison between the input signal and potentials at various tapping points of the voltage ladder established by the resistor string. Each comparator generates a ' 1 ' if $V_{I N}$ is larger than its associated reference level and a ' 0 ' if otherwise. The combined outputs of the comparators constitute a thermometer code, each of which represents a quantization level. An encoder then used to convert the thermometer code to a 3-bit digital output word. Encoder blocks have been implemented in several different ways. There are implementations such as the multiplexer based encoder [2], fat tree encoder, [8], and ROM encoder [5, 1]. The encoder scheme adopted this design was aimed at efficient code conversion, reduction of errors arising from metastability and also low power budget compliance.

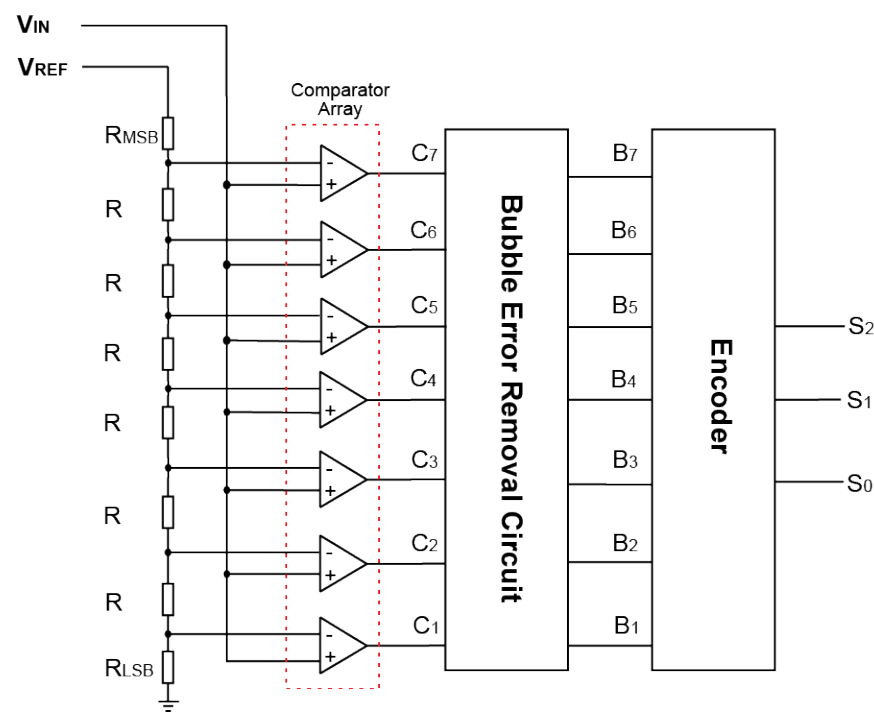

Fig. 1: Architecture of Flash ADC

Table 1 shows the design specification for the proposed ADC. The operation of ADCs typically involves the approximation of an analog input signal with a corresponding analog output in order to achieve conversion of the signal to a digital word. These approximations results in several errors like the quantization error. For low resolution converters, there exists a strong correlation between the input signal and the quantization noise. Apart from the quantization error, static noise like the deviation of actual quantization levels from ideal levels exist in non-ideal 
analog-to-digital converters. These result in both linear as well as non-linear deviations in the transfer curve of ADCs with offset and gain error being typical quantifications of linear deviations. Comparators switch instantaneously when their two inputs, $V^{+}$ and $V^{-}$achieve equal potential. This behaviour is however altered with the presence of offset voltage, $V_{O S}$. Outputs of nonideal comparators therefore experience state switches under the following conditions:

$$
\begin{aligned}
& V_{O}=1 \text { when } V^{+}>V^{-}+V_{O S} \\
& V_{O}=0 \text { when } V^{+}<V^{-}+V_{O S}
\end{aligned}
$$

Flash ADCs which have their reference voltage levels generated through resistive ladder typically use equal valued resistors for this purpose. The accuracy of quantization levels in this case relies on the degree of matching of the resistors in the string. The comparator voltage input on the $j_{t h}$ tap of the resistor ladder is the sum of an ideal voltage term and another consisting of the product of the converter's quantization step and the aggregate of resistor mismatch ratios. Mathematically, the $j_{t h}$ voltage tap is given as:

$$
V_{j}=V_{j, \text { ideal }}+\frac{V_{R E F}}{2^{N}} \cdot \sum_{k=1}^{j} \frac{\Delta R_{k}}{R}
$$

The ideal voltage term, $V_{j, \text { ideal }}$ represents the voltage that would be measured at the $j_{t h}$ tap should no resistors deviate from their nominal values, $R$ whiles $\Delta R_{k}$ represents the resistance mismatch error value. From Equation 3 , it is evident that the sum of the $\Delta R_{k}$ terms influences the magnitude of each voltage at tap and the converter linearity substantially. The integral nonlinearity, $I N L$, which defines the linearity of the comparator's overall transfer curve can therefore be estimated using Equation 4. The effects of resistor mismatches are also evident in alterations in the switching point of the comparators. Equation 5 estimates the switching voltage of the converter's $j_{t h}$ comparator.

$$
\begin{gathered}
I N L=V_{o s, j}+\frac{V_{R E F}}{2^{N}} \sum_{k=1}^{j} \frac{\Delta R_{k}}{R} \\
V_{s w, j}=V_{j}+V_{o s, j}
\end{gathered}
$$

\section{COMPONENT DESIGN}

\subsection{Comparator design}

The comparator circuit as shown in Figure 2 has three stages: an input preamplifier, a positive feedback and an output buffer. The preamplifier stage improves the comparator sensitivity by boosting weak input signals and shielding the comparator from kickback noise generated by the comparator's positive feedback stage. A fully differential transconductance amplifier with active PMOS loads $M_{3}$ and $M_{4}$, forms the input stage of the comparator. $M_{1}$ and $M_{2}$ are sized such that the resulting input node impedance is low in order to facilitate high bandwidth operations. The fully differential nature of the output of the first stage ensured that there was optimum rejection of common mode components of input signals. By choosing a suitable aspect ratio of the current mirrors, $M_{5}$ and $M_{6}$, these transistors upon acting on the first stage output signal, imparts a current gain. The drain currents, $I_{X}$ and $I_{Y}$ are related to the preamplifier's input voltages by

$$
I_{X}=\frac{g_{m}}{2}\left(V^{+}-V^{-}\right)+I_{S S}=I_{S S}-I_{Y}
$$

The comparator's decision making circuit was implemented using positive feedback because this configuration has the capability to distinguish $\mathrm{mV}$-level signals. Noise from sources such as the interfacing digital circuit as well as ADC's power supply were reduced in the feedback circuit by the addition of some amount of hysteresis. Assuming $I_{X}$ is much larger than $I_{Y}$ such that transistors $M_{7}$ and $M_{9}$ were on whilst $M_{9}, M_{1} 0$ were off, $V_{Y}$ is approximately $0 \mathrm{~V}$ and the value of $V_{X}$ is determined by Equation 7 where $\beta_{A}=\beta_{7}=\beta_{10}$.

$$
V_{X}=\sqrt{\frac{2 I_{X}}{\beta_{A}}}+V_{T H N}
$$

An increase and corresponding decrease of $I_{Y}$ and $I_{X}$ values respectively raises the gate overdrive potential of $M_{10}, V_{O V 10}$ to $0 \mathrm{~V}$; a condition which commences switching. Further changes in the currents in similar fashion causes $V_{O V 10}$ to realize positive values. $M_{8}$ as a result drains current away from $M_{7}$ decreasing $V_{D S 7}$ and $V_{D S 8}$; and switching $M_{9}$ off. At the switching point, the drain currents of $M_{9}$ and $M_{7}$ are respectively

$$
I_{X}=\frac{\beta_{A}\left(V_{X}-V_{T H N}\right)^{2}}{2}
$$

and

$$
I_{Y}=\frac{\beta_{B}\left(V_{X}-V_{T H N}\right)^{2}}{2}
$$

The current in $M_{9}$ mirrors that in $M_{7}$ at this instant, $I_{X}$ can therefore be seen as directly proportional to $I_{Y}$ related by a constant of $\frac{\beta_{A}}{\beta_{B}}$. Switching therefore occurs when the $\beta$ s are equal and the two currents attain equal values. The comparator, however exhibits hysteresis when $\beta_{A} \neq \beta_{B}$. Switching point voltages in this case are related to the input transistor transconductance, $g_{m}$ and differential pair tail current, $I_{S S}$ by:

$$
V_{S P H}=-V_{S P L}=v_{p}-v_{m}=\frac{I_{S S}}{g_{m}} \cdot \frac{\frac{\beta_{B}}{\beta_{A}}-1}{\frac{\beta_{B}}{\beta_{A}}+1}, \text { for } \beta_{B} \geq \beta_{A}
$$

The preamplification stage is loaded with the low impedance diode connected $M_{7}-M_{9}$ transistor pair whiles the cross connected gate $M_{8}-M_{10}$ pair serve as latch-forming logic inverters which constitute the decision element. The inverter pair transistors are sized such as to achieve a voltage gain larger than unity bearing in mind that they are also loaded by $M_{7}$ and $M_{10}$. Positive feedback from the cross-gate connection was used to increase the gain of the decision element and improve the output state transitions significantly. Typically, comparators tend to produce multiple output transitions when processing slowly-varying signals with even the slightest amount of superimposed noise. This back-and-forth bouncing occurs as a result of the amplification of noise as these signals transition the threshold region. To remedy this issue, some level of hysteresis is introduced into the decision element by choosing different $\beta$ 's in the design. The fractional feedback of the output voltage adds a polarity sensitive offset to the input thereby increasing the threshold range. The output buffer forms the third and final stage of the comparator. This post-amplifier stage converts the output of the second stage into one of two states: $V_{D D}$ or $V_{S S}$, thereby producing a logic signal. It is a desired characteristic of the output buffer that it is able to accept a differential input signal and exhibit little or no slew-rate limitations. In this design, a self-biased singleended differential amplifier was employed as the post-amplifier. An inverter was connected to the output of the differential amplifier to provide an additional gain stage and also help isolate any load capacitance from the self-biasing differential amplifier. The diode connected transistor, $M_{15}$ acts as a level shifter to move the decision circuit's output swing into the common-mode range of the differential amplifier. 


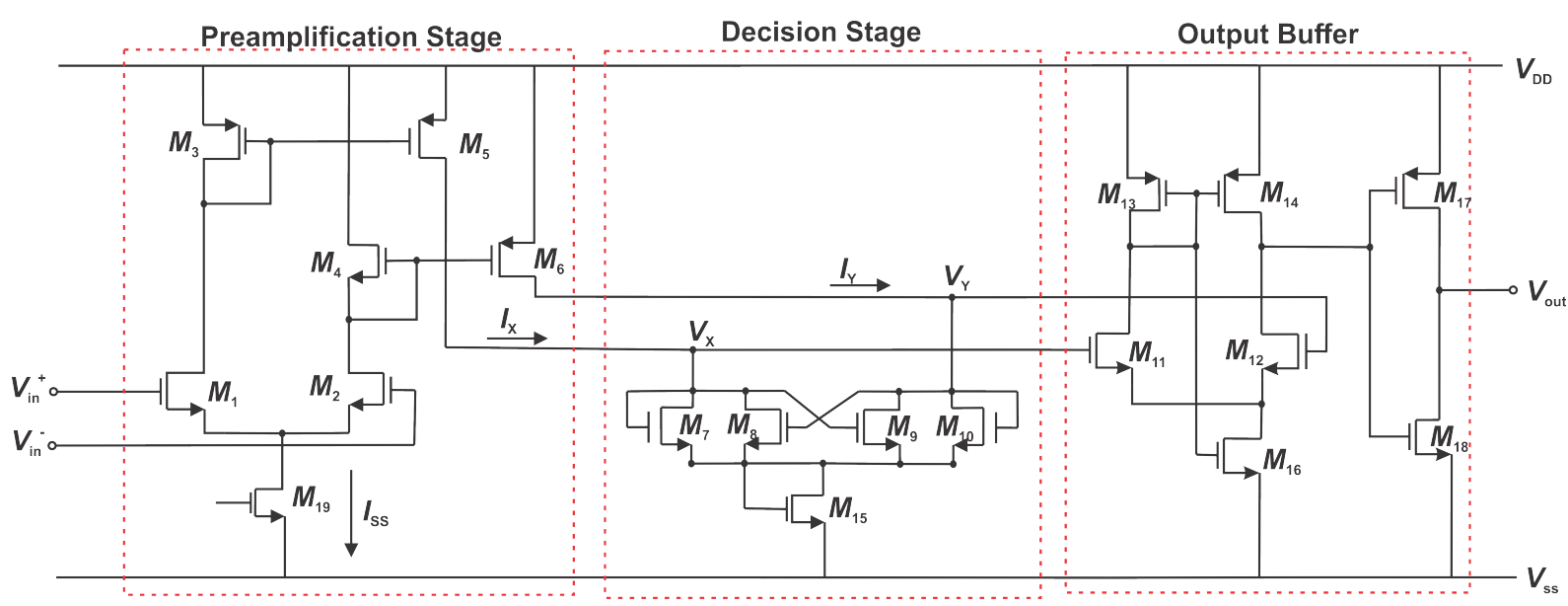

Fig. 2: Proposed comparator design

\subsection{Resistor ladder design}

The expected power consumption of the resistive ladder was estimated at $78.4 \mu \mathrm{W}$ in a worst case scenario. By having 8 resistors in the string, the value, $R$, of each resistor was determined from as:

$$
R=\frac{V_{R E F(M I N)}}{78.4 \mu \times 8}
$$

where $V_{R E F(M I N)}$ is the minimum allowable reference voltage obtained from the comparator design. Voltage quantization involves the approximation of the input signal given a set of fixed reference signals. Unlike Nyquist sampling, which is immune to errors, approximations in quantization introduce noise that cannot be removed [10]. For converters with very low resolution there is a strong relationship between the quantization error and the input signal [9]. Designed as mid-tread type quantizer, the 'staircase' transfer characteristic is shifted by $0.5 L S B$ so that code transitions occur around $L S B$ values and the quantization error centred about $0 L S B$. To achieve this, the values of the $R_{M S B}$ and $R_{L S B}$ were adjusted from to $1.5 R$ and $0.5 R$ respectively.

\subsection{Encoder design and bubble error correction}

The binary encoder designed to convert the 7-bit comparator output to the 3-bit digital word, included a bubble-suppressing logic to remove imperfections from the thermometer code. The bubble-error removal circuitry, which is inserted between the output of the comparators and the input of encoder, has seven 3 -input NAND logic gates connected as shown in Figure 3 With this introduction, the logic of encoding is modified such that only the presence of two consecutive ' 1 ' logic states right before ' 0 ' is indicative of a transition of thermometer code states. The logic table in Table 2 represents the original and revised comparator binary outputs in relation to the expected encoder output word. The Boolean expression that relates the expected output bits to the bubble-suppressing logic output bits are:

$$
\begin{aligned}
& S_{2}=\overline{B_{4} B_{5} B_{6} B_{7}}=\overline{B_{4} B_{5}}+\overline{B_{6} B_{7}} \\
& S_{1}=\overline{B_{2} B_{3} B_{6} B_{7}}=\overline{B_{2} B_{3}}+\overline{B_{6} B_{7}} \\
& S_{0}=\overline{B_{1} B_{3} B_{5} B_{7}}=\overline{B_{1} B_{3}}+\overline{B_{5} B_{7}}
\end{aligned}
$$

Table 2. : Truth table of the 3-bit binary encoder and bubble removal $\operatorname{logic}$

\begin{tabular}{|c|c|c|}
\hline $\begin{array}{c}\text { Comparator } \\
\text { Array Output } \\
C_{7} C_{6} C_{5} C_{4} C_{3} C_{2} C_{1}\end{array}$ & $\begin{array}{c}\text { Bubble-Removal } \\
\text { Circuit Output } \\
B_{7} B_{6} B_{5} B_{4} B_{3} B_{2} B_{1}\end{array}$ & $\begin{array}{c}\text { Expected } \\
\text { Encoder Output } \\
S_{2} S_{1} S_{0}\end{array}$ \\
\hline 0000000 & 1111111 & 000 \\
\hline 0000001 & 1111110 & 001 \\
\hline 0000011 & 1111101 & 010 \\
\hline 0000111 & 1111011 & 011 \\
\hline 0001111 & 1110111 & 100 \\
\hline 0011111 & 1101111 & 101 \\
\hline 011111 & 1011111 & 110 \\
\hline 1111111 & 0111111 & 111 \\
\hline
\end{tabular}

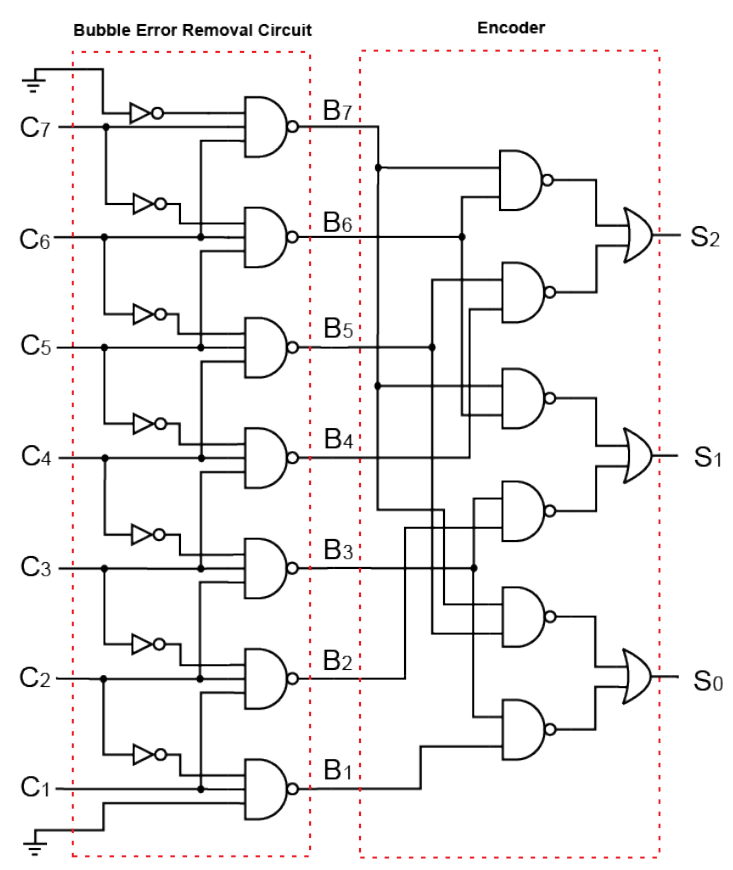

Fig. 3: 3-bit encoder with bubble removal circuit

The implementation of the encoder is shown in Figure 3 


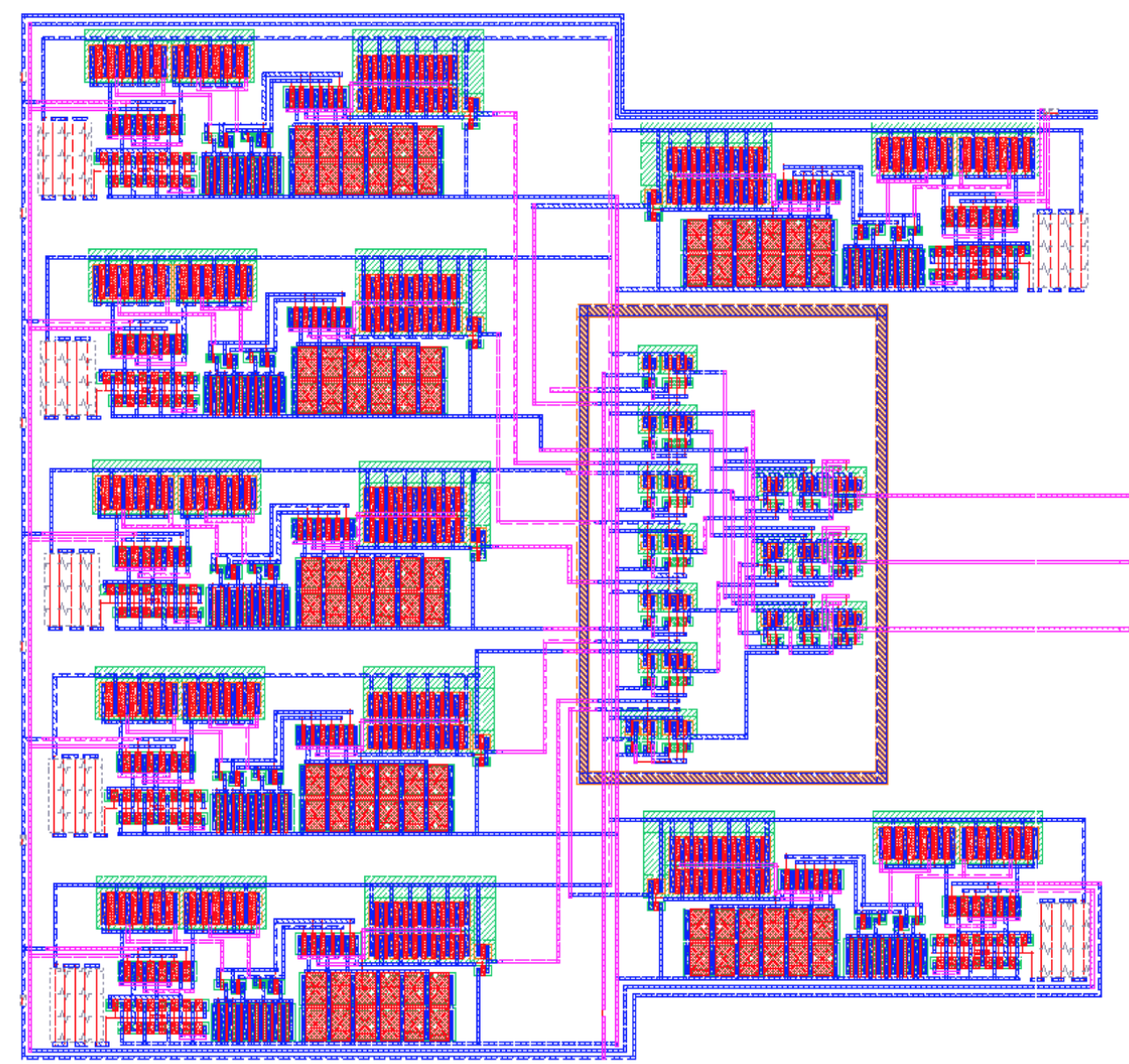

Fig. 4: Layout of the flash ADC in AMI $0.6 \mu \mathrm{m}$ technology

\subsection{Layout}

The flash converter layout was realized in the AMI $0.6 \mu \mathrm{m}$ technology is shown in Figure 4 The layout has an area of $343 \times 312$ $\mu \mathrm{m}^{2}$. Guard rings were placed around the digital circuit to shield the analog circuitry from substrate and power supply noise.

\section{SIMULATION RESULTS}

The extracted layout of the flash ADC was simulated using the Cadence Spector engine using a rail-to-rail voltage supply of $3 \mathrm{~V}$. The power dissipated in each comparator was $3.4 \mathrm{~mW}$ making the comparator array of 7 comparators dissipate a total power of approximately $23.79 \mathrm{~mW}$. The open-loop response of the converter shown in Figure 5 shows a DC gain is approximately $60 \mathrm{~dB}$ and an operating frequency of $2.17 \mathrm{MHz}$. Transient simulation of the comparator showed that the minimum differential input voltage detectable by the comparator is $34.13 \mathrm{mV}$. The post-layout comparator simulation metric results are summarized in Table 3

Table 3. : Post-layout simulation results for each comparator

\begin{tabular}{|l|l|}
\hline Parameter & Value \\
\hline Resolution & $34.13 \mathrm{mV}$ \\
\hline Slew Rate & $88.43 \mathrm{~V} / \mathrm{nS}$ \\
\hline Propagation Delay & $3.21 \mathrm{~ns}$ \\
\hline Sampling Rate & $10 \mathrm{MSps}$ \\
\hline
\end{tabular}

The output of the comparators are shown in Figure 6 The input signal is a ramp voltage signal with the comparator showing the desired output thermometer code. The minimum switching voltage occurred when $V_{I N}=17.25 \mathrm{mV}$ and the last occurred at $V_{I N}=227.5 \mathrm{mV}$. Table 4 shows the various transition voltages for each comparator.

Table 4. : Switches voltages for comparator stack

\begin{tabular}{|c|c|c|}
\hline$V_{I N}(m V)$ & $C_{7} C_{6} C_{5} C_{4} C_{3} C_{2} C_{1}$ & $S_{2} S_{1} S_{0}$ \\
\hline $0 \leq V_{I N}<17.25$ & 0000000 & 000 \\
\hline $17.25 \leq V_{I N}<52.5$ & 0000001 & 001 \\
\hline $52.5 \leq V_{I N}<87.5$ & 0000011 & 010 \\
\hline $87.5 \leq V_{I N}<122.5$ & 0000111 & 011 \\
\hline $122.5 \leq V_{I N}<157.5$ & 0001111 & 100 \\
\hline $157.5 \leq V_{I N}<192.5$ & 0011111 & 101 \\
\hline $192.5 \leq V_{I N}<227.5$ & 0111111 & 110 \\
\hline $227.5 \leq V_{I} N$ & 1111111 & 111 \\
\hline
\end{tabular}

The output of the ADC in response to the ramp and a sinusoidal waveform are shown in Figure 7

Table 5 summarizes a comparison of the post-layout performance simulation results of the designed Flash ADC to some existing works. 


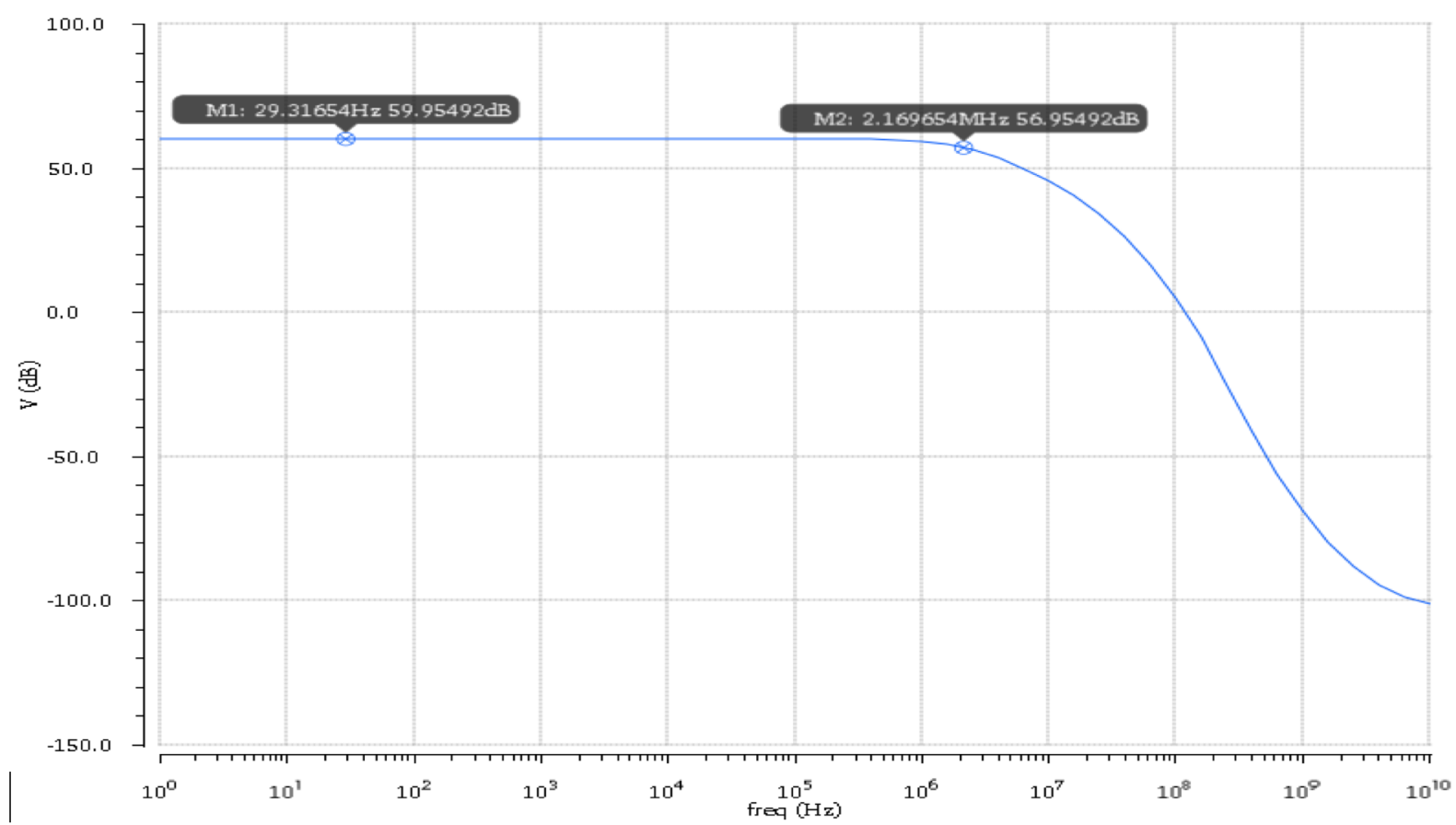

Fig. 5: Frequency response of ADC showing a dc gain of $60 \mathrm{~dB}$ and a cutoff frequency of over $2 \mathrm{MHz}$.

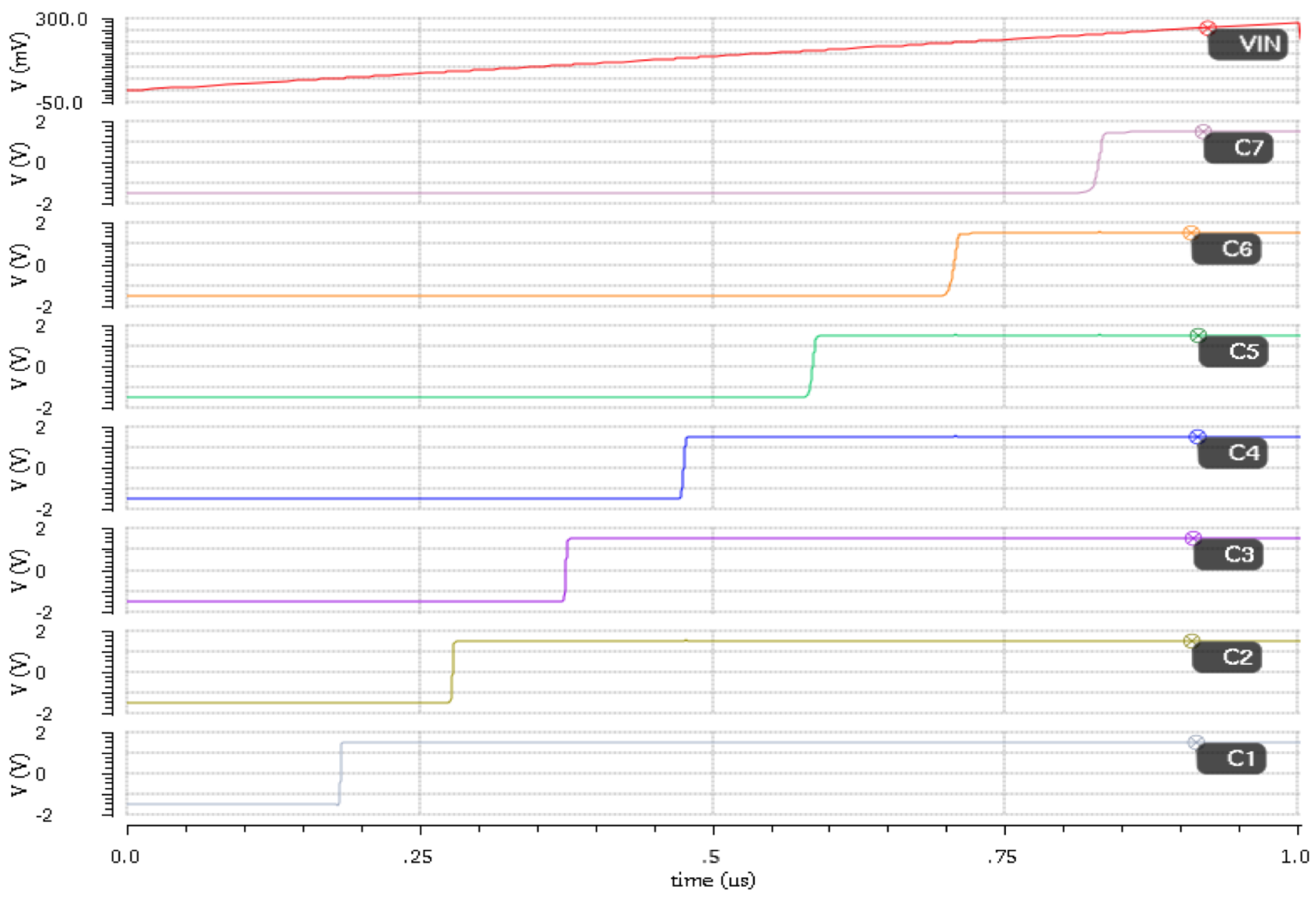

Fig. 6: Output of Comparator with a ramp input voltage 


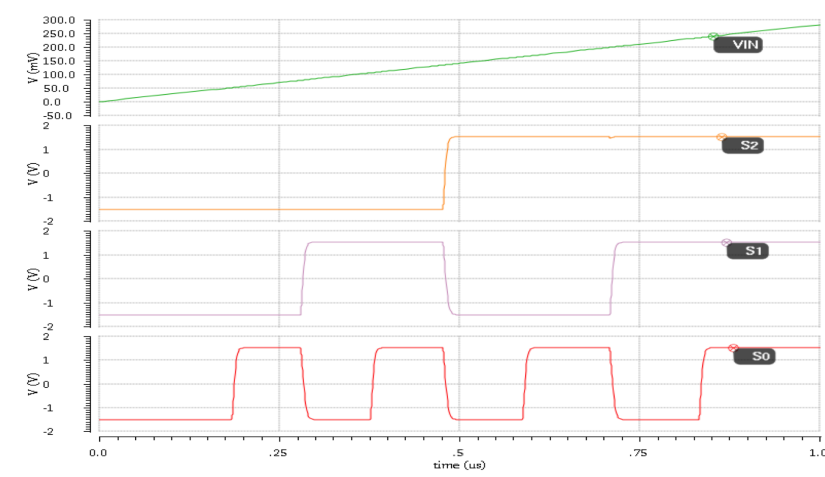

(a) ramp input signal

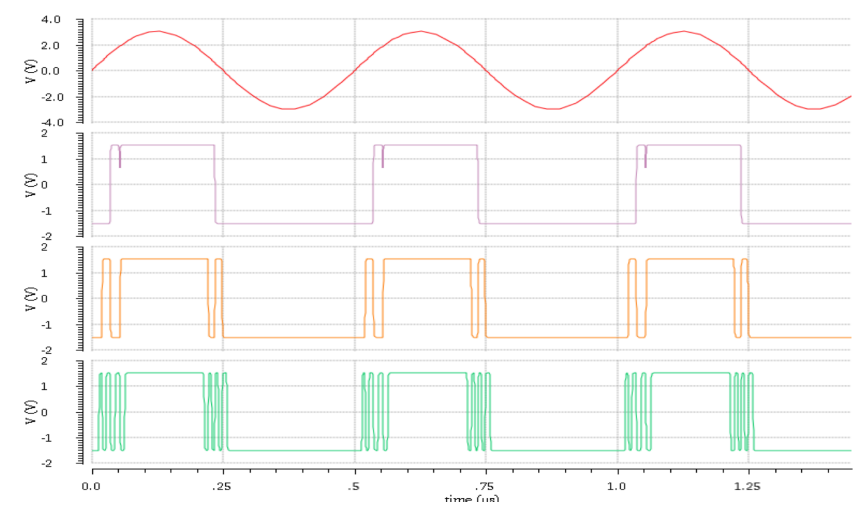

(b) sine input signal

Fig. 7: Output of the ADC with different input signal waveforms

Table 5. : Comparison of results

\begin{tabular}{|c|c|c|c|c|}
\hline Parameter & {$[3]$} & {$[4]$} & {$[11]$} & This work \\
\hline Technology & $180 \mathrm{~nm}$ & $45 \mathrm{~nm}$ & $180 \mathrm{~nm}$ & $600 \mathrm{~nm}$ \\
\hline Resolution & 3 -bit & 3 -bit & 3-bit & 3 -bit \\
\hline Supply voltage & $1.8 \mathrm{~V}$ & $0.7 \mathrm{~V}$ & $1.3 \mathrm{~V}$ & $3 \mathrm{~V}$ \\
\hline Speed & - & - & - & $10 \mathrm{MSps}$ \\
\hline Power consumption & $19.47 \mu \mathrm{W}$ & $218 \mu \mathrm{W}$ & $36.237 \mathrm{~mW}$ & $23.88 \mathrm{~mW}$ \\
\hline Bandwidth & $10 \mathrm{MHz}$ & $5 \mathrm{MHz}$ & $20 \mathrm{MHz}$ & $2.17 \mathrm{MHz}$ \\
\hline
\end{tabular}

\section{CONCLUSION}

In this paper, a low power 3-bit flash ADC is designed and its performance simulated. The above work outlines the design of the

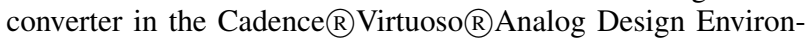
ment and the device simulation using Spectre $\cap$ circuit simulator in the AMI06 CMOS technology. The design featured a resistive ladder, three-stage comparators, a bubble noise suppression circuit and an encoder. Simulation results for the design showed a low device power dissipation of $23.7 \mathrm{~mW}$, an input frequency of $2.17 \mathrm{MHz}$ and a conversion speed of $10 \mathrm{MSps}$ achieved with a $3 \mathrm{~V}$ supply. Results also showed that the transition voltages were a bit different from what was calculated. This was due to the time delay of the comparators and the digital circuit in propagating the signal. The non-ideality introduced into the ADC by adding hysteresis could also be a factor. The designed converter can be used for low power and high speed applications.

\section{REFERENCES}

[1] N. Agrawal and R. Paily. An improved rom architecture for bubble error suppression in high speed flash adcs. In 2008 Annual IEEE Student Paper Conference, pages 1-5, Feb 2008.

[2] Mark Vesterbackar Erik Sail. A multiplexer based decoder for flash analog-to-digital converter. In IEEE region 10 TENCON conference, volume 4, pages 250-253, Nov 2004.

[3] Pradeep Kumar and Amit Kolhe. Design \& implementation of low power 3-bit flash adc in $0.18 \mu \mathrm{m}$ cmos. International Journal of Soft Computing and Engineering (IJSCE), 1(5):71-74, 2011.

[4] Swati Mishra, Abhay Vidyarthi, and Shyam Akashe. A novel folding technique for 3 bit flash adc in nanoscale. In Advanced Computing and Communication Technologies (ACCT), 2013 Third International Conference on, pages 307-311. IEEE, 2013.

[5] M. Rahman, K.L. Baishnab, and F.A. Talukdar. A novel rom architecture for reducing bubble and meta-stability errors in high speed flash adcs. In 20th International Conference on Electronics Communications and Computers, pages 15-19, 2010.

[6] P. V. Rahul, A. A. Kulkarni, S. Sankanur, and M. Raghavendra. Reduced comparators for low power flash adc using tsmc018. In 2017 International conference on Microelectronic Devices, Circuits and Systems (ICMDCS), pages 15, Aug 2017.

[7] B. Razavi. The flash adc [a circuit for all seasons]. IEEE Solid-State Circuits Magazine, 9(3):9-13, Summer 2017.

[8] Akashe Shyamm et al. Optimization of fat tree encoder for ultra high speed analog-to-digital converter using 45 nanometer technology. Optic-International Journal for Light and Electron Optics, 124(20):4490-4492, 2013.

[9] Timmy Sundstrom and Atila Alvandpour. A 2.5-gs/s 30mw 4-bit flash adc in 90nm cmos. In NORCHIP, 2008., pages 264-267. IEEE, 2008.

[10] Rudy J Van de Plassche. CMOS integrated analog-todigital and digital-to-analog converters, volume 742 . Springer Science \& Business Media, 2013.

[11] Amana Yadav et al. Design and implementation of low power 3-bit flash adc using $180 \mathrm{~nm}$ cmos technology. International Journal of Engineering Research and Applications, 6(6):77-82, 2016. 